\title{
DU TRANSPORT DES BLESSÉS EN MONTAGNE
}

Dans une conférence faite le 3 février 1906 'aux officiers de la place de Lyon, sur le service de santé dans la guerre de montagne, M. Dubrulle, médecin principal de deuxième classe à l'hôpital militaire Villemanzy ${ }^{1}$, après avoir passé en revue les conditions du transport, les formations sanitaires et leur service pendant les marches et au combat, le fonctionnement des hôpitaux de campagne, concluait par les données suivantes, en ce qui concerne le transport des blessés :

a Le chargement sur roues du matériel sanitaire des corps de troupes ne mérite aucune confiance. Les voitures médicales régimentaires ne peuvent circuler en dehors des routes. Il conviendra de leur substituer des animaux de bât,... qu'il serait avantageux d'étendre à toutes les troupes de montagne.

" L'effort demandé aux brancardiers excèdera souvent les limites de la résistance physique. Il faudra qu'après l'engagement, un certain nombre de combattants laissent le fusil pour contribuer au relèvement des blessés.

( Le brancard ne convient pas toujours pour le transport du point de chute au poste de secours. Il y aura lieu parfois de le remplacer par le transport à dos d'homme, ou par des appareils improvisés avec les ressources locales.

"Le relai d'ambulance, très peu distant des postes de secours, sera formé par des groupes de brancardiers, de cacoleis et de litières, auxquels on adjoindra tous les moyens de fortune de la région. IJes blessés graves seront placés sur litières, sur traîneaux de ferme ou sur brancards traînés suivant le système Eybert.

a Le traînage paraît très recommandable en ce qu'il offrirait au patient plus de confort et de sécurité. Peut-être deviendra-t-il le procédé de choix destiné à supplanter les précédents pour l'enlèvement des hommes les plus grièvement atteints. "

Nous renvoyons le lecteur à la remarquable conférence du Dr Dubrulle en ce qui concerne l'organisation du personnel, l'installation de l'ambulance et des hòpitaux de campagne, par lesquels,

1 Archives de médecine et de pharmacie militaires. $\mathrm{N}^{0} 8, \mathrm{p} .113$. 
soit dit en passant, il recommande le morcellement, rendu encore plus nécessaire en montagne qu'en plaine.

Nous ne retiendrons ici que les conclusions de lauteur en ce qui concorne le transport en montagne, qu'un remarquable mémoire du même périodique, dủ à $\mathrm{M}$. Paul Eybert, médecin-major de $2^{\text {me }}$ classe aux hôpitaux de la division d'occupation de Tunisie, vient développer plus loin avec une réelle compétence.

(Ce travail intitulé: Etude sur le transport des blessés en montagne et sur le brancard alpin a le mérite d'avoir été fait sur le terrain même, de relater des expériences vécues, très judicieusement observées el commentées.

Nous ne pouvons donner ici qu'un résumé très sommaire de ce mémoire dont nous recommandons l'étude dans l'article original ${ }^{1}$. Les expériences de M. Eybert nous paraisgent applicables à toutes les éventualités de la guerre en pays montagneux et éclairer d'un jour nouveau les conditions du relèvement et du transport des blessés dans ces circonstances. Rien n'est oublié par l'auteur, ni les conditions du recrutement du personnel brancardier, ni le matériel à disposition, ni les mille difficultés du transport.

A examiner les choses de près à cet égard, un premier point doil fixer l'attention des services sanitaires, c'est que le transport des blessés est chose laborieuse qui exige beaucoup de temps. Si l'on considère que les brancardiers ne sont pas recrutés généralement parmi les hommes les plus forts, le rendement obtenu par les moyens actuels est faible, on peut dire nettement insuffisant.

«Deux brancardiers portant un blessé, dit M. Eybert, sont en général, capables de marcher dix minutes à un quart d'heure, sans arrêt et de repartir après deux minutes de pause. L'allure est, à peu près, dans ces conditions, de 4 kilomètres à l'heure si les difficultés du terrain sont moyennes. "En examinant les conditions résultant des différences de niveau du terrain à franchir, $M$. Eybert conclut que: «deux hommes mettront ainsi deux heures et demie pour en relever un autre, et si l'on évalue à $5 \%$ de l'affectif total le nombre des blessés à évacuer, les huit équipes d'un bataillon évacueront les 25 blessés d'une unité de 500 hom-

${ }^{1}$ Archives de médecine et de pharmacie militaires. 1906, no 9, p. 197, avec de nombréuses figures. 
mes en sept heures et demie à huit heures. "Et encore ce résultat exige t-il de la part des brancardiers un travail continu qui avoisine le surmenage, encore les conditions du relèvement ne sont-elles pas toujours aussi favorables.

Il résulte de ces faits que la question des moyens de transport doit être serrée du plus près possible, de manière à en améliorer les conditions.

L'auteur passe en revue les différentes ressources à disposition sur le terrain : Le transport à dos d'hommes est très fatigant et doit être réservé pour des circonstances spéciales. M. Eybert n'a jamais pu obtenir des brancardiers, par ce moyen, plus de dix minutes de marche sans un essoufflement considérable qui oblige à des temps de repos prolongés: "Impressionné, ajoute-t-il, par l'idée que ce mode de transport a pu paraître pratique en Suisse, où la sellette est règlementaire et jouirait d'une certaine faveur auprès des camarades des corps alpins, nous nous sommes attaché à faire vérifier autant que possible ces résultats et à réaliser, pour ainsi dire, des essais publics : dans chaque expérience exécutée en quelque sorle sous le contròle de nombreux officiers, le rendement a été déplorable, même après vingt-cing jours de marche et d'entrâ̂nement suivi. "

Examinant toutefois de plus près les conditions de ce transport, M. Eybert constate qu'en répartissant la charge à la fois sur les lombes, les épauleset le front (qui met en jeu les muscles de la nuque), on facilite la tâche du brancardier; encore faut-il qu'il soit vigoureux et:transporte un blessé léger. A cet égard la meilleure sellette serait peut-être la plus simple aussi, c'est la capote du porteur, le pan en étant fortement relevé sur le dos du blessé et les coins antérieurs roulés, passant sous les bras du blessé, et se réfléchissant sur l'épaule du porteur qui les tient à pleines mains. Le blessé, enveloppé et maintenu moeileusement sans compression, depuis les jarrets jusqu'à l'aisselle, ne peut balloter ni tomber. C'est un peu la méthode employée par les femmes africaines pour transporter leurs enfants sur le dos.

Autre chose estule transport par brancard ; el encore le brancard règlementaire, relativement commode en terrain plat, devient-il, à la descente, un supplice pour le blessé qui glisse constamment, ainsi que pour les porteurs, surtoul celui d'arrière, qui, 
ne sachant où il met les pieds, tombe fréquemment et durement. L'auteur a imaginé pour obvier à ce double inconvénient un dispositif qui assimile plus ou moins le brancard de montagne à la Stuhlbahre de Port et permet au brancardier d'arrière, placé entre les jambes relevées et écartées du blessé, de voir son chemin. Cette modification au brancard est insignifiante et peu coûteuse et alteint parfaitement son but.

L'auteur a cherché aussi à améliorer la sangle qui passe sur les épaules des porteurs et fatigue beaucoup la nuque jusqu'à la meurtrir en peu de temps.

Ainsi le simple évidement de l'espace sis entre les jambes du blessé, l'adoption d'une barre de réflexion pour les jarrets et le relâchement de la toile, avec la bricole-collier de M. Eybert, constituent un brancard de montagne très pratique. "Nous avons fait constater, dit l'auteur, que ce dispositif une fois réalisé permet de passer facilement partout, même dans les parties boisées de la montagne, que la fatigue des brancardiers est réellement moindre, que le confort du blessé ne laisse rien à désirer, en un mot que le rendement est maximum. " L'auteur l'évalue, en chiffres, à $30 \%$.

Mais M. Eybert insiste encore sur une autre amélioration capitale dans le mode de transport, c'est l'organisation de relais de distance en distance pour les brancardiers. De la sorte le transport s'opère sans arrêt et chaque équipe se repose avant de remonter ou de redescendre. La montée et la descente font travailler des groupes de muscles différents et, fait qui a sa valeur, les brancardiers se familiarisent plus vite avec les obstacles rencontrés dans le chemin suivi plusieurs fois de suite. En outre la création de relais permet pour chaque section du trajet l'utilisation de l'appareil de choix : sur telle section on sera forcé de transporter à dos, sur telle autre, avec le brancard à deux, ou par traînage, ou sur bête de somme.

"C'est la seule façon, dit M. Eybert, d'obtenir le rendement maximum. »

Nous avons eu l'occasion de voir transporter récemment, d'après ce principe, par une équipe d'une cinquantaine d'hommes, le matériel disparate, très lourd et encombrant, d'un refuge alpin complet, boiseries, toiture, mobilier, etc., sur des chemins tantôt abrupts, tantòt vertigineux, à travers les crevasses d'un grand gla- 
cier, le long des pentes raides de roches escarpés, sur une dis. tance de plus de 6 heures de route et le tout en peu de jours.

Chaque équipe avait son secteur et, après quelques transports, les hommes étaient si habitués aux difficultés du trajet parcouru et à parcourir, qu'elles devenaient pour eux, chose en apparence négligeable; en outre, le retour à vide après des trajets de 200 à 500 mètres, suivant le terrain, réalisait, après chaque effort, un repos de quelques minutes et un changement d'allure. A aucun moment nous n'avons vu ces hommes, obligés pourtant à des efforts considérables et à un travail sans relâche, présenter le moindre symptôme de lassitude, et le rendement du travail, comparé aux méthodes habituelles des transports analogues, a été sans doute doublé comme rapidité.

Ce mode de transport oblige, toutefois, en ce qui concerne les blessés, à des transbordements fréquents et éventuellement pénibles. M. Eybert a cherché à y obvier par la construction très ingénieuse d'un support unique pouvant s'adapter aux différents genres de transports. Nous y reviendrons plus loin.

Pour ce qui concerne les brancards improvisés, l'auteur insiste sur l'importance capitale qu'il y a à ce que l'étude des moyens à employer ait été faite d'avance fréquemment et minutieusement et que le mot d'improvisation implique aussi règlementation. Le général $D^{r}$ Port avait déjà insisté sur ce point et il est incontestable que la connaissarice théorique la plus parfaite d'un procédé d'improvisation peut laisser en plan, au moment du besoin, l'opérateur non entraîné par des exercices pratiques répétés. A cet égard l'infirmier improvisé reste un piètre improvisateur. On ne saurait l'oublier dans les sociétés charitables où les aides de la dernière heure, en cas de guerre ou de calamités publiques, sont plus souvent des embarras que des secours.

Pour :improviser le brancard il faut un matériel élémentaire, sans doute, mais qui manque souvent dans la montagne. Pour y obvier, l'auteur reprend une idée émise par le $D^{r}$ Frœlich, celle de dépôts de perches, cordes, marteaux, clous, etc., dans différents chalets et cabanes alpestres, de même que dans les approvisionnements des voitures médicales des troupes de montagne. Le brancard alpin recommandé par l'auteur, composé de deux perches de 2 mètres, de deux traverses, de la toile des tentes et de quelques 
cordes, pèse à peine $10 \mathrm{kilos}$, souvent moins, donc à peine plus que le brancard règlementaire à hampes pliantes.

En pays de montagne, l'auteur a eu à se louer des brancards traînants « qui permettent, dit-il, de supprimer le porteur d'arrière, le rôle de celui-ci restant borné à retenir le brancard au moyen d'une corde pour éviter les chutes et les heurts, aider aux passages difficiles et soulager le porteur d'avant. Ces brancards, ajoute-t-il, facilitent la descente sans surmener les brancardiers et sont aisés a improviser, ils peuvent, sans transborder le blessé, être attachés à l'arrière d'une charrette ou d'un mulet de bât déjà chargé ; enfin le confort y est très grand et nous avons souvent joui, ajoute M. Eybert, de l'étonnement de camarades, faisant office de blessés, im. pressionnés par avance des difficultés de la descente rocailleuse et finalement convaincus des avantages de ce mode de transport. " Ce traînage permet une allure parfois fort rapide, si la pente est suffisante, le pas de gymnastique peut être employé sans effet désagréable pour le patient.

Naturellement le brancard traînant perd ses avantages si la pente est faible et dans ce cas, il faut qu'il puisse être porté à deux. On peut en improviser ne dépassant pas le poids de dix à douze kilos, ce qui n'excède pas de beaucoup le poids du brancard normal.

L'auteur a procédé à des essais exacts pour apprécier mathématiquement la part de poids supporté par les brancardiers dans les différentes positions du malade et des porteurs. Il en résulte tout d'abord que c'est du côté de la tête du blessé que doit être toujours placé le brancardier le plus robuste et que, même à la descente, la pente influe dans une proportion moindre sur le poids supporté par le porteur que le sens du blessé. Dans le trainage il y aurait donc théoriquement avanlage, pour soulager le porteur, à mettre le blessé tête arrière, mais par contre la traction est nettement plus dure; ce qu'on gagne sur le poids porté, on le perd en frottement.

(Il n'en reste pas moins acquis, dit l'auteur, que le porteur d'un brancard traînant ne porte pas sensiblement plus que dans le cas du brancard ordinaire porté à deux, mais il doit fournir un effort de traction, variable suivant la pente, aussi la fatigue est plus prompte, le travail fourni étant plus considérable. Par contre le blessé traîné est descendu plus vite que le blessé porté et les brancar- 
diers se relayant ainsi plus fréquemment ne se fatiguent pas plus, en fin de compte.

L'auteur a fait la inême constatation pour les animaux de bât: " un mulet, préalablement chargé de deux blessés sur un cacolet, ne s'aperçoit pas du brançard traînant qu'on y ajoute. Il n'est même pas nécessaire que ce harnachement comporte une bricole, ce qui indique que le tirage est insignifiant par rapport au poids déjà porté; de simples attaches de cordes de chaque côté du bât suffisent. )

Il va sans dire, que pour ce mode de transport, lit barre de réflexion pour les jambes du blessé « système Stuhlbahre » est nécessaire, au risque de le voir glisser. Tant qu'on n'a pas atteint le chemin carrossable, il est utile qu'un brancardier surveille l'arrière et, au besoin, soulève par endroits le traîneau. Sur des chemins meilleurs non seulement cette surveillance devient inutile, mais on peut ajouter, à l'ensemble, un second traîneau, attelé au premier, de façon que les deux blessés soient superposés, sans se toucher d'ailleurs, de la sorte le rendement est très grand puisque le mulet se trouve porter quatre hommes, deux assis sur les cacolets el deux couchés sur les brancards traînants. "A noter, toutefois, ajoute M. Eybert dont le coup d'œil pratique n'oublie rien, que la pouszière soulevée recouvre rapidement le blessé le plus inférieur. »

Ces essais ne sont pas théoriques, M. Eybert a effectué plusieurs cols élevés avec des chargements de ce genre, le mulet ayant ainsi pu faire dix kilomètres avec un chargement maximum sans fatigue anormale ni blessure quelconque. Inutile d'ajouter que la même disposition s'obtient à l'arrière d'une charrette; sur de bonnes routes on peut mettre deux blessés côte à côle avec trois perches seulement comme syslème de traînage.

L'auteur conclut que le traînage peut, sur certaine parcours, doubler le rendement tout en nécessitant moins de personnel. Il estime qu'il est donc appelé à rendre de très grands services en montagne.

Passant au transport par bêtes de somme, M. Eybert estime que le cacolet a fait ses preuves partout ailleurs qu'en montagne ou un cerlain discrédit a souvent empêché de l'utiliser. Il est évidemment inconfortable, angoissant pour le malade par les balan- 
cements considérables, émotionnant sur certains chemins par les glissements de la bête, les secousses, etc.

L'auteur considère toutefois ce moyen comme suffisamment sùr, dans la majorité des cas, pour des blessés légers; il est souvent du reste d'un emploi inévitable, s'il s'agit de mener rapidement une évacuation. Il estime par contre que, dans le transport d'un seul homme sur l'animal à nu, ou sur un bât, ou sur une selle même, le confort est moins grand que sur le cacolet, et plus dangereux pour un blessé grave sujet à une syncope ou à une faiblesse. L'au. teur mentionne aussi, comme convenant à certains cas, la litière attelée entre deux mulets marchant l'un devant l'autre, du type employé universellement chez presque tous les peuples de la terre avant l'invention des voitures.

L'utilité du bât pour le transport des blessés couchés a inspiré à M. Cadiot, médecin-major de $2^{\text {me }}$ classe, l'invention d'un appareil pliant, fort ingénieux et facilement transportable, qui, accroché des deux côtés du bât, vient supporter très solidement et aussi confortablement que possible le brancard posé transversalement sur le dos de la bête. Le balancement doit sans doute être considérable mais la sécurité est complète et ce système pourra rendre de réels services ${ }^{1}$.

Il n'existait pas, jusqu'ici, d'appareil permetlant le transport aussi bien à dos d'homme que par brancard avec deux ou plusieurs porteurs, ou à dos de bêtes, ou par voitures. M. Eybert a été tenté par ce problème et propose la solution suivante: Il fait un brancard composé de deux hampes, non pliantes, et de deux traverses; pas de charnière, pour assurer la solidité et la légèreté; la traverse inférieure est placée au niveau des jarrets ; les jambes sont maintenues dans des gouttières dont le croissant supérieur, matelassé convenablement, fait l'office de la traverse de réflexion des jarrets dans la Sluhlbahre; la toile, peu tendue pour recevoir le siège du malade, est fixée à ces croissants, fendue au milieu sur 40 centimètres de longueur et munie d'œillets permettant de la tendre à volonté. Les deux bords de la fente sont relevés entre les cuisses et attachés à la hampe correspondante; on évite ainsi le glissement. Ce brancard, qui rappelle la Stuhlbahre de Port, ne

' Archives de médecine et de pharmacie militaires, $1906 \mathrm{n}^{\circ}$ 9, p. 239. 
pèse que 5 kilos, moins donc que la sellette et que le brancard alpin. Il ne comprend que quatre boulons pour toutes ferrures et s'est montré très résistant à l'usage pendant de longs mois d'emploi.

On comprend d'emblée comment il peut être adapté au transport à dos d'homme, avec, ou mieux, sans traverse de réflexion, le malade étant à cheval sur les lombes du porteur, soutenu par la toile el les hampes du brancard el au besoin, par une bricole passée sur le front ou sur l'épaule du porteur. Celui-ci peut de la sorte avoir, s'il le faut, les mains libres et s'aider de son alpenstock.

Pour le transport à deux, le brancard est porté, au moyen des bricoles, comme le brancard ordinaire. A mulet il peut être fixé latéralement au hât, comme le cacolet, et présente beaucoup moins d'inconvénients que ce dernier, le balancement à la marche étant moins sensible et les secousses très atténuées. On peut, de même, l'employer pour le transport axile pour un seul blessé, dans des conditions de sécurité suffisantes. Toutefois M. Eybert déconseille avec raison ce mode de transport : Q Qu'il s'agisse d'un blessé peu grave pouvant reposer par son siège sur un bât de faible épaisseur, c'est faisable; mais qu'il faille coucher un blessé grave au-dessus du bât règlementaire, on ne peut éviter de surélever trop la charge qui oscille... et d'exposer aux coups de tête du mulet; le tangage et le roulis sont pénibles, le chargement difficile ». L'auteur recommande donc le cacolet pour deux blessés, ou bien, si le chemin est trop étroit pour cela, de faire monter à cheval un blessé peu grave, en adjoignant au bât un brancard traînant où les conditions de sécurité et de confort son t bien mieux remplies. Cet attelage descend tous les sentiers; aux lournants il suffit de soulever l'arrière du trainean; dans les cas dilficiles on peut le dételer momentanément.

L'auteur traite ensuite du transport par voitures. Il voudrait voir adopter dans les voitures réglementaires des ressorts de suspension pour les hampes des brancards, vu la durelé des cahots dans ces roitures un peu massives. Même observation pour les voitures de blessés improvisées dont l'aménagement est du reste toujours facile avec des perches et des cordes.

Les traîneaux à bras des Alpes, servant à transporter le bois en hiver, peuvent rendre d'excellents services; l'auteur propose un mode d'adaptation du brancard alpin modifiéqui assure une meil- 
leure position du blessé. "Ces ramasses ou traineaux à bras se trouvent, dit-il, en grand nombre dans les chalets habités du Briançonnais, la plupart pèsent dix kilos au plus ; un mulet remontant à vide peut en amener quatre ou cinq sur le lieu de l'action. C'est donc un moyen d'évacuation de tout premier ordre qu'il fau ${ }^{-}$ dra toujours utiliser même près de la ligne de feu. Nous lui avons trouvé, ajoule-t-il, tous les avantages du traînage, d'autant mieux que, plus bas, sur bonne route, même plate, on peut atteler ces traîneaux les uns aux autres et organiser ainsi de véritables trains avec un minimum de personnel. 》

Gette question du traîneau-brancard pour le transport des malades a fait l'objet d'une étude détaillée due à la plume de M. Gauthier, médecin-major de $2^{\text {me }}$ classe au $12^{\text {me }}$ bataillon d'artillerie à Briançon. M. Gauthier a réussi à réaliser par ce moyen une couchette très confortable pour le blessé et d'un maniement facile pour le transport. La suspension du brancard y est excellente et absolument sûre ${ }^{1}$.

Le mémoire de M. Eybert, dont nous n'avons pu donner qu'un aperçu incomplel, conclut en constatant combien le problème du transport en montagne est complexe ; l'improvisation elle-même, qui doit rendre tant de services, a besoin d'être étudiée à l'avance, règlementée et modifiée éventuellement suivant des circonstances bien définies. Le choix des brancardiers est chose de toute importance aussi ; ils doivent être vigoureux ; leur instruction, forcément négligée, de par les règlements qui ne permettent pas leur dressage journalier sur un terrain approprié, a lieu d'être modifiée. L'art de relever un blessé, pense M. Eybert avec raison, est trop utile et trop difficile en même temps pour que les 40 à 50 séances annuelles affectées à cette instruction soient suffisantes.

Il est en effet de toute importance que ce genre d'enseignement tout d'à propos et d'expériences vécues, soit fait dans les conditions qui se rapprochent le plus de celles d'un véritable combat en montagne. Il est de valeur à peu près nulle s'il n'est pas fait sur le terrain. Et, pour ce qui nous concerne, cet axiome est aussi vrai pour l'infirmier volontaire que pour le brancardier militaire; cela va de soi, et, à cel égard, personne ne trouvera son instruction

${ }^{1}$ Archives de médecine et de pharmacie militaire, 1906, n. 9, p. 250. 
trop perfectionnée. Mais il n'est pas donné à chacun de savoir donner cet enseignement avec le sens pratique et, nous voudrions dire, le génie de l'auteur du mémoire que nous venons d'analyser.

C'est ce qui nous a engagé à consacrer à ce mémoire une place un peu plus étendue que nous n'en avons l'habitude dans nos analyses; le Iravail de $M$. Eybert est de ceux qui font faire un pas en avant dans les questions complexes et difficiles de l'improvisation des secours.

Dr FerRiÈre.

\section{GRANDE BRETAGNE}

LA HUITIÈME GONFÉRENCE INTERNATIONALE DES SOCIÉTÉs DE LA CROIX-ROUGE

Circulaires du Comité de la Conférence aux Sociétes de la Croix-Rouge.

Octobre 1906.

Monsieur le Président,

J'ai l'honneur de vous rappeler, au nom de notre Société que, conformément à une proposition du Comité international de la Croix-Rouge, qui a élé portée à votre connaissance par une circulaire no 114, en date du 20 mars dernier, la huilième Conférence internationale des Sociétés de la Croix-Rouge doit avoir lieu à Londres au cours de la semaine commençant le 10 juin 1907.

Notre Société a été très sensible à l'honneur que lui a fait le Comité international, en choisissant son pays comme siège de la prochaine Conférence, el elle se félicite baaucoup d'avoir pu l'accepter avec le gracieux assentiment de LL. MM. le roi Edouard et la reine Alexandra.

Nous serions heureux que vous voulussiez bien prendre part à cette Conférence, et si vous acceptez notre invitation, nous vous serions obligés de nous faire connaître le plus tòt possible les noms de vos délégués.

Vous voudrez bien nous communiquer également dans le plus 